\title{
Exhibiting the Effects of the Episodic Buffer on Learning with Serial and Parallel Presentations of Materials
}

\author{
Eshaa M. Alkhalifa \\ Royal University for Women, West Riffa, Bahrain
}

\author{
eshaa@ieee.org
}

\begin{abstract}
The effectiveness of an informing system is based upon several factors that include the perceptual limitations of the person receiving the information. This paper examines the perceptual limitation of the amount of information that may be processed by the human cognitive system when this information is displayed in parallel through multiple windows. The experiments show that a sequential presentation of information is more effective than a parallel one in information transfer of large amounts of information or highly complex information in cognitively demanding subjects like mathematics. These conclusions are informative to educational system designers of complex subjects.
\end{abstract}

Keywords: working memory limits, multimedia system design, computer based learning, perceptual limitations, student modeling.

\section{Introduction}

Informing systems, in general, are systems that attempt to maximize the amount of information transferred from a computer system to the memory of the human being informed by that system. The efficiency of the information transfer is affected by the limitations and characteristics of the human cognitive system. Indeed, a vast amount of research was directed toward human memory in order to identify the factors that may stimulate recall of information.

In particular, short-term recall of events in the world exhibits characteristics distinct from longterm recall. While long-term recall is believed to be unlimited, short-term recall is severely limited. Miller (1956) showed that people face a difficulty in memorizing and recalling long strings of numbers.

This led researchers to investigate short-term memory further in order to identify its limitations and, by consequence, their influence on the perception of information. Baddeley and Hitch (1974) found that presenting spatial information on a screen while a subject performs a spatial

Material published as part of this publication, either on-line or in print, is copyrighted by the Informing Science Institute. Permission to make digital or paper copy of part or all of these works for personal or classroom use is granted without fee provided that the copies are not made or distributed for profit or commercial advantage AND that copies 1) bear this notice in full and 2) give the full citation on the first page. It is permissible to abstract these works so long as credit is given. To copy in all other cases or to republish or to post on a server or to redistribute to lists requires specific permission and payment of a fee. Contact Publisher@InformingScience.org to request redistribution permission. task causes interference, while presenting acoustic information does not affect subject performance in the task. They claimed that these results reflect that the memory space reserved for verbal and spatial information is distinct from the memory space reserved for auditory information.

Moreno and Meyer (2000) build on this by defining a split-attention (parallel) 
principle. Instead of having subjects perform a task while textual information is displayed on a screen, they split the screen into two windows: textual and animated. Interference was detected when subjects were closely monitored for time and disappeared when Tabbers, Martens, and Van Merrieboer (2001) gave them unlimited time. In retrospect, these results reflect a restriction represented as limited attention showing that a person cannot attend to the parallel windows within that limited time frame. The lessons presented by Moreno and Mayer (2000) may be considered simple concepts that do not challenge an average student. If, on the other hand, complex learning materials are used then the difference in learning between parallel and sequential presentation may re-appear. Consequently, if learners are allowed free time to learn complex concepts and continue to show a limitation in learning then another cause for the difference may exist. Forms of complexity include the cognitive demands of a problem on a learner's cognitive system (Gill and Hicks, 2006).

Schnotz and Kürschner (2007) indicate that working memory, by definition, has to hold the current problem state, the goal state, any subgoal states as well as any relations or operators continually in working memory until the task is accomplished. In a learning situation where a human has to hold all these states in memory, then, two possibilities exist. The first is that the learner has a scope of perception as wide as the limits of working memory. Multiple windows may be used in the informing system without any loss in the efficiency of the information transfer because as long as there is space in working memory then more information can be perceived. The second is that the perceptual system does not have a bandwidth equal to that of working memory. In this case, only a serial presentation of material is advised in highly complex information transfer domains. Do note that in this condition the memory size requirements of complex materials funnel through the bottleneck posed by perception's memory without overflowing the receiving working memory container. If the test material does overwhelm working memory, then no conclusions can be made between the two above possibilities.

This paper investigates the effectiveness of presenting the same amount and complexity of information to a problem solver in parallel on a screen compared with presenting it sequentially. If the serial presentation of information produces better results, then a bottleneck limits how much information may be received in parallel. This would be consistent with an assumption that the total memory size of short-term memory is larger than the bandwidth of parallel information transfer. Working memory accordingly may store and later processes the information without any effort while keeping all problem states in memory. If, on the other hand, the parallel presentation learning results are equivalent to the serial presentation, then the only size limit imposed on the information perceived is the overall size of short-term perceptual memory.

This paper's findings support the existence of a limitation in the bandwidth and also support the existence of a distinct episodic buffer used for synthesis of information. The paper starts by reviewing relevant work to present indicators of the existence of the limited bandwidth in existing literature. It presents the approach followed to test the phenomenon's existence and explore it. That is followed with a discussion of the impact of these findings on informing system design that should take these cognitive restrictions into account.

\section{Background}

Miller (1956) was one of the first who described in print the limitations of working memory. He described the short term memory's limits of seven chunks representing seven information blocks that can be remembered at one time. If information can be represented in a form that is more semantically related to prior knowledge or that is composed of no more than seven blocks, then it is more likely to be remembered. For example, telephone numbers of length seven may be recalled as a sequence of individual numbers, while numbers of length eight are usually recalled in blocks of two or more. 
The existence of this limitation in short term memory encouraged researchers to examine its impact on perceiving information. A central assumption is that human perceptual devices are directly connection to a perceptual memory. What ears hear, for example, is immediately recorded as acoustic information in working memory. Eyes, by contrast, record printed verbal and pictorial information into working memory. Investigating the characteristics of this perceptual memory consequently involved examining interference in perception.

Baddeley and Hitch (1974), for example, discovered that presenting subjects with spatial information on a screen distracts subjects, causing them to make more errors in a spatial activity based task. On the other hand, hearing the auditory version of the same information did not have the same results.

Based upon these findings, Baddeley and Hitch (1974) proposed a model for short-term memory that assumes it is composed of at least three parts (Baddeley, 1998, 2000, 2003a, 2003b). The model defines working memory as composed of a central executive which controls the other two modules through limited attention, a phonological loop that holds and manipulates acoustic and speech based information, and a visuospatial sketchpad that is responsible for visual information as shown in Figure 1.

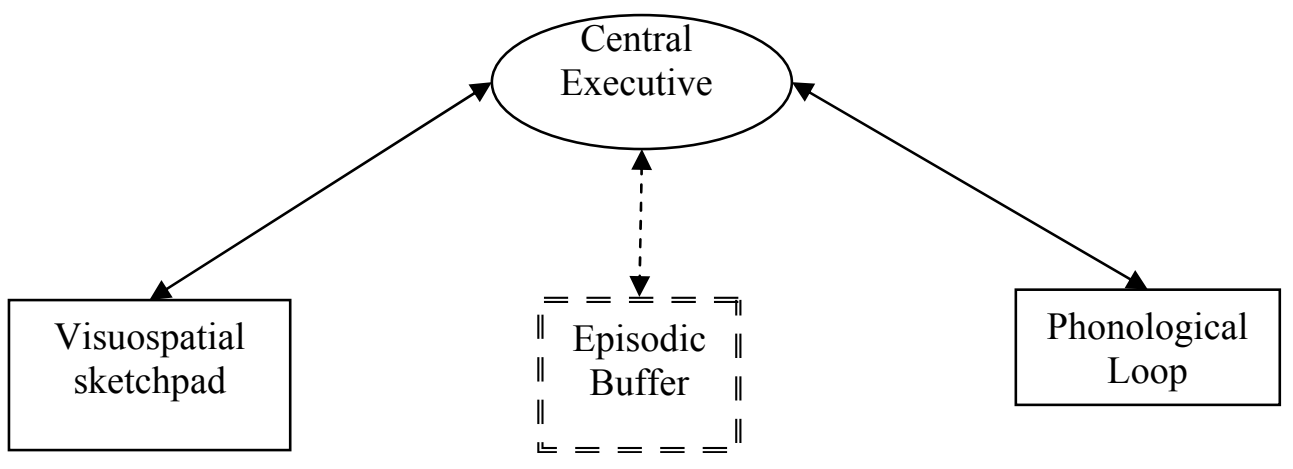

Figure 1: The elements originally proposed by Baddeley and Hitch (1974) and in the dashed lines the element recently suggested by Baddeley (2000).

Current theory indicates that the phonological loop is composed of two components. The first is a passive memory store that holds memory only for a few seconds as it decays, and the second component is a subvocal rehearsal system that refreshes the information stored as well as registers object names within the store if a visual object was perceived and can be named. The visuospatial sketchpad is primarily responsible for what is perceived visually, like color/shape, or spatially, as in items shown above or below other items.

The episodic buffer, however, is a memory that was introduced as a result of several challenges to the three part structure. An extremely intelligent patient who had an impaired long term memory showed normal immediate memory for passages of prose of up to 25 idea units, which is well beyond the assumed capacity of the phonological loop or the visuospatial sketchpad. These idea units required some memory space to hold them until the subject was asked to repeat them, and that memory has to be a form of working memory. The episodic buffer is assumed to play a role in the interaction between the loop and the sketchpad by presenting itself as a common memory (under the control of the central executive) for the merging of spatial and verbal information, for example, the naming of images. It should not, however, have any direct interaction with the outside world since it is not part of the phonological loop, which receives verbal and auditory information from perception, nor is it part of the visual and spatial perceptual system. 
An additional issue is consciousness of the environment, which is already implicitly assumed by the original model because it defines a way of receiving and storing information from the environment. The central executive can attend to a specific event that is stored in the episodic buffer presenting a form of conscious thought. Last but not least the episodic buffer is limited in nature and not unlimited as in the Long Term Memory to which it provides an interface.

This is informative to information system designers because the model interprets empirical results by emphasizing the main characteristics of working memory. The model reveals that some media when mixed, like text and animation, require more processing time from the client to attend to all the information, while media like animation and audio mix well and do not have that extra requirement.

\section{How Complex is Complexity}

Gill and Hicks (2006) offer an analysis of complexity classes of task or problems a system user has to face from a large reference pool. They conclude that existing complexity definitions fall into five main classes.

1. Objective Complexity: Under this class complexity is measured as strictly dependent on the characteristics of the task itself. A task that requires two pages of problem solving differs in complexity from a task that asks problem solvers to add two positive integers.

2. Lack of Structure Complexity: The degree of organization in a task to be performed affects its complexity. Performing a search for a name in a sorted list has a lower level of complexity than searching in an unsorted list.

3. Problem Space Complexity: This type of complexity depends on the characteristics of a problem space. One question may have more than one solution with one solution much easier than other possible solutions. This type of difference is usually evident in mathematical proof type problems where one approach to the proof may require a lower number of steps than other approaches to solve the same problem.

4. Information Processing Complexity: On the one hand, it is possible for problem solvers to face a problem that requires complicated mathematical formulas to solve. On the other hand, it is possible to have a problem that only requires basic common knowledge information to solve.

5. Experienced Complexity: Problem solvers who are acquainted with the testing procedures followed by a specific instructor are more experienced and have lower anxiety levels prior to a test by that instructor than problem solvers who are taking their first course with an instructor.

The first two classes of complexity are solely dependent on the problem, while the latter three rely on cognitive processing required to solve the problem. The third depends on a problem solver's ability to compare different possible solutions with each other simultaneously while the fourth requires more information manipulation in a possibly long solution.

Consequently, complexity may be directly related to cognitive load. Increasing the amount of information a problem solver has to hold and process in memory is equivalent to increasing the cognitive load requirements. Cognitive load theory (Sweller, 1988; Sweller, Chandler, Tierney, \& Cooper, 1990) defines cognitive load to refer to any demands on working memory storage and processing of information. Working memory, by contrast, refers to the structures and processes used for temporary storage and manipulation of information as opposed to long term memory where memories are kept for a long duration of time. In other words, informing systems present 
information that is processed in working memory and later stored in long term memory. This implies that limitations of working memory are of high relevance to informing systems.

Cognitive load was later split up to describe the additive sum of intrinsic load, extraneous load, and germane load (Sweller, 1988; Sweller et. al., 1990; Sweller, van Merrienboer, \& Paas, 1998). Intrinsic load is the load that is required by the nature of the task itself, while extraneous load represents the unnecessary load that is required by the presentation approach that is utilized when presenting the task to learners. Germane load is a recent addition to the above two and describes a positive load effect that increases load requirements of the learning task in a positive fashion (Sweller, 2005).

Therefore, intrinsic load is the minimum memory processing requirement by a specific task. Extraneous load, on the other hand, is load that is not necessary, usually caused by the approach selected to present the material. Information can be presented through static screens, through an interactive problem solving session, through a multimedia display, etc. Each one of these will have different memory requirements; for example, a hypermedia linked document expects the reader to recall the pages they linked from and to, while reading a book does not expect readers to recall the pages they read prior to the current one. Germane load is an additional load requirement to memory that aids in remembering learning of the presented information.

In other words, when considering Problem Space complexity from a cognitive perspective, the total number of possibilities that a learner has to recall may include hypermedia address information of various resources, which may be described as germane load, in addition to the problem states required to arrive at a solution (intrinsic load). A poorly defined informing system may also require a learner to recall how to search and find information (extraneous load).

This paper investigates a cognitive trait that may have a direct influence on the level of complexity faced by the learner utilizing an informing system.

\section{Investigating the Existence of a Perception Based Bottleneck}

Although the initial presentation of the episodic buffer in 2000 by Baddeley was accompanied by justifications for its introduction, it has not been subject to empirical investigation. Consequently, the work presented here aims to investigate the consequences of its existence. It is by definition larger in size than the memories of the phonological loop and the visuospatial sketchpad. Therefore, the total capacity of working memory is expected to exceed what is perceived at a specific point in time by both perceptual memories.

Since the buffer is distinct from the visuospatial sketchpad and the phonological loop, investigative materials should imply both representations. This is necessary to require the memory of the learners to use the buffer as a support for the two memories available. Mathematics was chosen for this role because, although the presentation is through verbal descriptions in lessons, it is found to include spatial representation in more than one research finding (Gevers, Reynvoet, \& Fias, 2003; Guay \& Daniel, 1977). Findings indicate that ordinal sequence ordering of numbers is spatially organized, which implies that this material will invoke the visuospatial sketchpad as well as the phonological loop that will deal with the verbal encasing of the presented educational lesson. This fits the goals of the experiment to make it necessary to use the buffer because it is the only part of working memory that is capable of assimilating the information presented verbally and the information represented with the sketchpad. Findings may be extended to represent any topic that invokes both working memory modules with equivalent complexity requirements. 


\section{Challenging the Capacities of the Loop and Sketchpad}

In order to test the existence of the buffer, the amount and complexity of educational material that is presented should be larger than the capacity of the memories in the loop and sketchpad. Since the buffer exists in the background, the memories of the loop and sketchpad will present an obstacle to all information obtained from the outside. This dictates that if the educational material is presented in parallel, the loop and sketchpad should be overwhelmed and cause a reduction in learning. On the other hand, if the same amount of educational material is presented serially then this educational material will be first stored in the loop and sketchpad and then filter to the episodic buffer, which is by definition larger in capacity. The existence of the buffer could then be tested by exhibiting more effective learning caused by the serial presentation of materials than by a parallel presentation of the same amount of material.

This paper will present an experiment that will expose problem solvers to two different settings to compare the effects of presenting parallel educational materials versus presenting sequential educational materials of the same size to identify if a working memory buffer exists in the background without direct access to the perceptual world. The paper will also examine the degree of similarity that exist between the pre- and post-tests. Questions of the same class of operations will be designed such that they have high similarity, intermediate similarity, or low similarity. The pre and post test questions that will be matched are division, multiplication and power. If the question in the pre test has a division operation that is $1 / 2$ and the post test has a $1 / 3$ then these two questions have a high similarity rating because the difference from pre and post test is in only one digit. Lower similarity ratings will mean that the difference is in more than one digit or location of the digit. Problem solvers may try to recall the question types they came across in the pretest to focus more on the learning materials that will benefit their post test performance. Results are expected to show that they will focus on all three operations that are present in the pre-test and show a positive improvement in the post test. If their results are not better in all three operations, then they did not pay extra attention to the operations in the pre-test and this attention does not explain their learning or lack of it.

\section{Designing the Medium of Investigation}

In order to compare the two conditions students were divided into two groups. One group was presented with the material arranged in a sequential manner while the other group was presented the materials such that they had to compare the information in different parts of the screen simultaneously. Pre- and post-tests were done to measure the effectiveness of each method while ensuring that these tests were set according to strict similarity ratings as is explained below.

The topic selected is geometric mathematical series of the format $1+2+3+4+\ldots+100$ that can also be represented as a mathematical notation with a summation sign and a starting and ending point as well as a variable to represent the change from one term to the next. The above example will therefore have the summation format:

$$
\sum_{\mathrm{i}=1}^{100} \mathrm{i}
$$

The term " $\mathrm{i}$ " in this case is a simple term but it may appear with a number that is added to it as in $\mathrm{i}+2$, or a number that multiplied as in $2 \mathrm{i}$ or even with a power (exponent) operation like $2^{\mathrm{i}}$ or $\mathrm{i}^{2}$. The operations addition or subtraction, multiplication or division, and the power operation are in rising degrees of complexity to the learner because they require increasing more computational processing. 
Since mathematical numbers are assumed to have a spatial representation (Gevers et al., 2003; Guay \& Daniel, 1977) this material seems ideal and with sufficient complexity to overwhelm the memory capacities of the phonological loop and visuospatial sketchpad. Schnotz and Kürschner (2007) indicate that working memory holds all the different states, from the initial states to the goal states, until the task is accomplished. It is, therefore, the aim of this experiment to overwhelm the working memory with tasks that require multiple states. These tasks are then presented either in parallel or in sequence to play the role of conceptual exercise blocks to measure the limits of the cognitive reception bandwidth. If the total capacity of working memory is larger than the reception bandwidth, which is limited by the sizes of the memories in the loop and sketchpad, then a serial presentation of the materials will be more effective than a parallel presentation. Working memory will still be able to hold all states while processing the task because a background working memory, which has been defined by Baddeley (2000) as the episodic buffer, does indeed exist.

If, on the other hand, the limit of working memory is similar to or controls reception bandwidth, then the parallel presentation will be no different than a serial one. Both presentation styles will require working memory to hold all states until all processing is complete and the approach used to receive the information will be subject to only perceptual limitations. There should be no difference in the amount of information that is presented serially from the amount presented in parallel to ensure that the comparison is a valid one.

The interactive tutoring system used to present information starts by explaining basic information to problem solvers and then presents them with an Interactive Module. They use this model by typing different numbers and watching while the system generates the series their numbers will generate. They are therefore capable of testing any form of question that is similar to or different from the ones they had to solve in the pre-test.

\section{Sequential Demonstration Module}

Since a primary concern of the tests run here is to identify if sequential presentation of information has a different impact from parallel presentation of information with the same total load requirements, problem solvers were tested before and after the sequential part of the educational system.

This is presented as a practice test session. Problem solvers answer seven questions in sequence. Participants see a mathematical series and problem solvers are expected to write the notation that may generate that series. When they write the notation, they can generate the resulting series from what they wrote. They can then compare the result of the generation to the series in the question. They can alter their notation and regenerate until they arrive at the notation that will generate the series in the question.

The questions are all accessed from an index page that displays a list of test question numbers, so learners can only review one question at a time sequentially. They are, therefore, not able to display more than one question on the screen at the same time. They are tested following this stage of learning using a post-test.

\section{Parallel Demonstration Module}

The second group of problem solvers completed the interactive learning phase then went on to the Parallel module.

The top half of the screen distinguishes the main components of the solution, which includes where a series starts, where it ends, and the notation that generates the terms. The second half of the screen displays the same components so that students have to compare the top of the screen to 
the bottom of the screen. The top part displays the ideal solution, alerting problem solvers to the correct starting and ending numbers, and the correct notation to associate with the series.

The second part uses an expert system that shows the probability of that student making a particular mistake and generates according to that probability how it expects that student to solve this new problem. In other words, it uses what it monitored as this student's technique in solving problems to solve this problem.

The information about that student is collected from the previous phase of interactive learning. The resulting series places the student in a teacher's shoes to compare the response to the ideal and to recognize where they went wrong. The main assumption in developing this approach is that it may cause students to recognize by comparison how to improve their approach to solving the problem.

A problem solver has to click on Problem 1 to see the fields in the top half of the screen filled. The series field will show the series that is part of the problem, and the other fields will show the corresponding starting and ending values in addition to the corresponding notation. Upon clicking View 1, the problem solver can see how errors previously made by that problem solver produce different results. The errors in the starting and ending values affect the length of the series that results, so the number of terms is different. Additionally, an error in writing the notation will result in a different looking series that is shown in series field of the View window. In Figure 2, a problem solver has made the error of multiplying the index $n$ by 2 instead of by 20 and replacing the starting and ending points by 10 to 19 instead of from 1 to 10 . Although the response seems to be a different approach to the problem, the resulting series is visibly very different from the one displayed in the Problem 1 series field. Figure 2 shows how the same problem is tackled twice, once with an ideal answer, and the second with some of the errors the same problem solver demonstrated in prior trials.

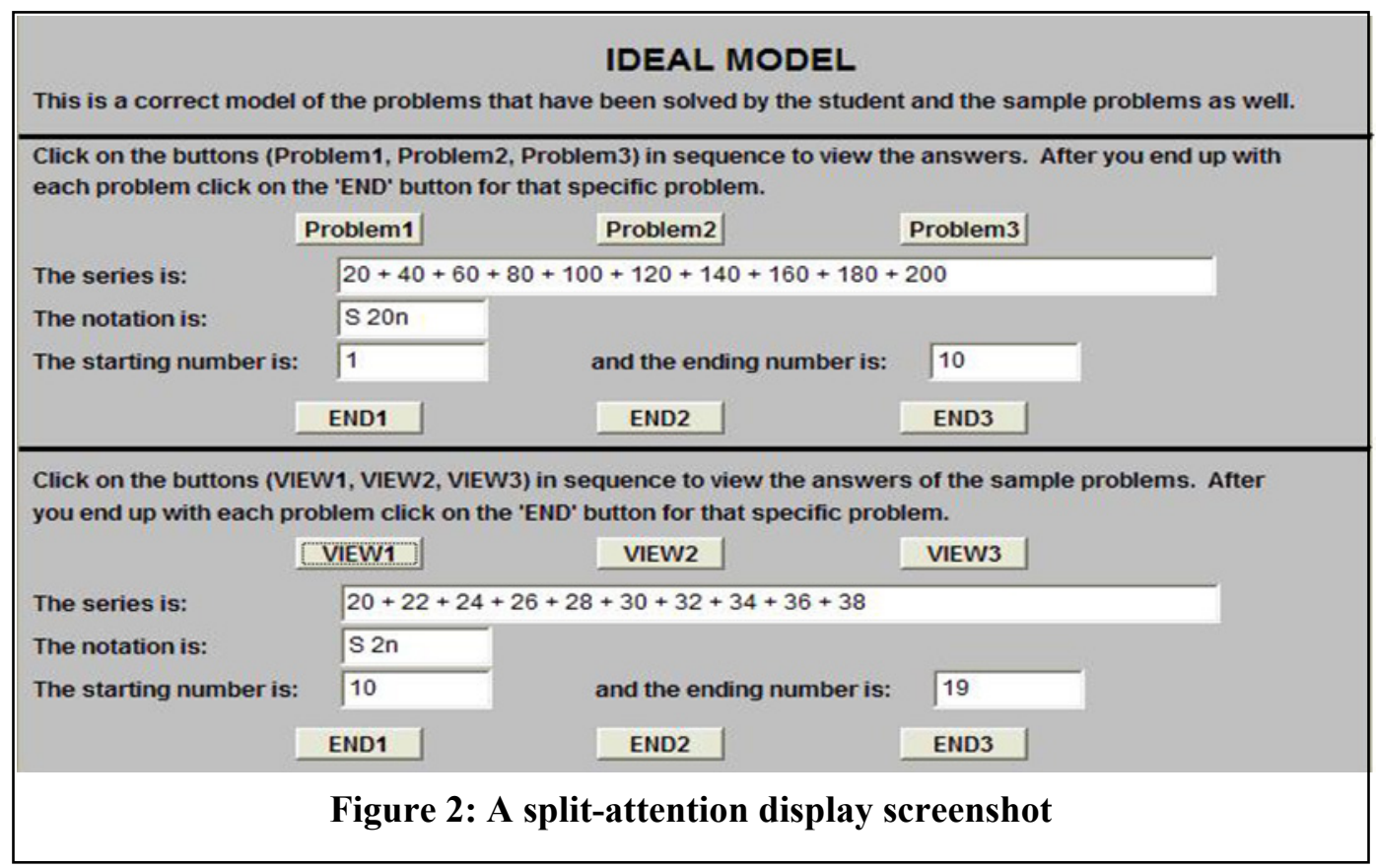

Consequently, a problem solver has to review two solutions each of Problem 1, Problem 2, and Problem 3 while comparing notations, starting points, ending points, and outcomes in order to 
learn how differences in each result in different series. This type of instruction is consequently described as parallel because at each instance of representing a problem, two different possibilities that are highly similar are displayed to show the effects of the differences between them. On the other hand, with the sequential presentation only one series and the correct response is displayed at any point in time, with more examples being displayed in total to ensure they cover all the possibilities. This group is also tested with a post-test.

\section{A Comparison of the Pre- and Post-Test Questions}

Problem solvers took a pre-test before using the tutorial system and post-test following its use to ensure that what is measured by the post-test results from exposure to the system and is not a result of prior knowledge.

Questions were selected based upon the main operation that generates the geometric series and these were division, multiplication, and the power operations. Marking is always out of 6 because a pilot study of this learning material revealed that six types of problem solver errors can be isolated as clearly as possible. These are:

- Error 1 The arithmetic operation in the chosen notation is incorrect.

- Error 2 The integer number in the notation is incorrect.

- Error 3 The starting number of the chosen notation is incorrect.

- Error 4 The ending number of the chosen notation is incorrect.

- Error 5 The number of terms in the resultant series of the chosen notation is less than the number of terms in the problem's series.

- Error 6 The number of terms in the resultant series of the chosen notation exceeds the number of terms in the problem's series.

Note that Errors 5 and 6 also depend on Errors 3 and 4, which implies that they are not completely independent.

The division pre-test question differed from the division post-test question by one digit, which is the value of the denominator and which qualifies division to have the highest similarity rating of pre- and post-test questions.

The multiplication question differed in the digit value multiplied and the digit value added to both the starting and ending numbers, which means a total of two differences. Consequently, it is at second place with regard to pre- to post-test similarity.

The power question differed in the alphabet letter used as a variable and had different increases in the values of the starting number and the ending number. Making the difference more pronounced. Therefore, this operation is regarded as having the least similarity between the pre- to post-test questions.

The prediction is that if the working memory buffer is episodic, then it is likely to be sensitive to the degree of similarity and that this should be reflected in the pre- to post-test data. Additionally, if all students did was to recall the pre test questions to search for the materials that explain how to solve them, then all results in post-tests for the three cases should show an improvement.

\section{The Evaluation Experiment}

The experiment was run over a four and half hour duration with two randomly distributed groups of problem solvers who completed the task in return for course credit. They were all third and fourth year students majoring in BSc computer science and Computer Engineering. The first group was composed of 32 students and the second group was composed of 16 students. 


\section{Results}

The results of the pre- and post-test of the sequential group are shown in Table 1. The last line on each table with italic text shows the average marks obtained by problem solvers out of 6 . Question 1 had a multiplication operation while Question 2 had a power operation and Question 3 had a division.

\begin{tabular}{|c|c|c|c|c|c|}
\hline \multicolumn{3}{|c|}{ Pre } & \multicolumn{3}{|c|}{ Post } \\
\hline Q1 & Q2 & Q3 & Q1 & Q2 & Q3 \\
\hline 2 & 1 & 2 & 2 & 2 & 1 \\
\hline 2 & 6 & 2 & 5 & 6 & 6 \\
\hline 2 & 6 & 2 & 5 & 6 & 6 \\
\hline 2 & 3 & 2 & 4 & 2 & 6 \\
\hline 2 & 2 & 2 & 6 & 3 & 1 \\
\hline 1 & 2 & 2 & 6 & 6 & 6 \\
\hline 2 & 3 & 6 & 6 & 6 & 6 \\
\hline 2 & 2 & 2 & 6 & 3 & 1 \\
\hline 2 & 5 & 2 & 1 & 1 & 6 \\
\hline 1 & 1 & 1 & 6 & 6 & 6 \\
\hline 2 & 3 & 6 & 6 & 6 & 6 \\
\hline 2 & 3 & 2 & 6 & 6 & 6 \\
\hline 2 & 6 & 6 & 4 & 6 & 6 \\
\hline 2 & 1 & 2 & 2 & 2 & 2 \\
\hline 2 & 3 & 2 & 4 & 2 & 6 \\
\hline 2 & 3 & 6 & 6 & 6 & 6 \\
\hline 2 & 3 & 2 & 6 & 6 & 6 \\
\hline 2 & 1 & 2 & 2 & 2 & 1 \\
\hline 1 & 1 & 1 & 6 & 6 & 6 \\
\hline 2 & 3 & 3 & 6 & 6 & 6 \\
\hline 2 & 3 & 3 & 6 & 6 & 6 \\
\hline 2 & 5 & 2 & 1 & 1 & 6 \\
\hline 2 & 1 & 3 & 4 & 4 & 6 \\
\hline 2 & 3 & 6 & 6 & 6 & 6 \\
\hline 2 & 1 & 2 & 2 & 2 & 2 \\
\hline 2 & 6 & 6 & 4 & 6 & 6 \\
\hline 0 & 0 & 1 & 1 & 1 & 6 \\
\hline 2 & 3 & 2 & 6 & 6 & 6 \\
\hline 2 & 3 & 2 & 6 & 6 & 6 \\
\hline 2 & 1 & 3 & 4 & 4 & 6 \\
\hline 1 & 2 & 2 & 6 & 6 & 6 \\
\hline 0 & 0 & 1 & 1 & 1 & 6 \\
\hline 1.75 & 2.69 & 2.75 & 4.44 & 4.31 & 5.13 \\
\hline
\end{tabular}


The results of the parallel group are shown in Table 2. The last line shows the average marks obtained by problem solvers out of 6 .

\begin{tabular}{|c|c|c|c|c|c|}
\hline \multicolumn{3}{|c|}{ Pre } & \multicolumn{3}{|c|}{ Post } \\
\hline Q1 & Q2 & Q3 & Q1 & Q2 & $\mathbf{Q 3}$ \\
\hline 6 & 6 & 1 & 6 & 6 & 6 \\
\hline 4 & 2 & 1 & 5 & 1 & 6 \\
\hline 4 & 2 & 1 & 5 & 1 & 6 \\
\hline 6 & 2 & 1 & 6 & 5 & 6 \\
\hline 6 & 5 & 6 & 6 & 3 & 6 \\
\hline 6 & 5 & 2 & 6 & 6 & 6 \\
\hline 6 & 5 & 2 & 6 & 6 & 6 \\
\hline 6 & 6 & 1 & 6 & 6 & 6 \\
\hline 6 & 6 & 6 & 6 & 2 & 6 \\
\hline 6 & 5 & 6 & 6 & 3 & 6 \\
\hline 6 & 6 & 1 & 6 & 2 & 6 \\
\hline 2 & 6 & 6 & 6 & 6 & 6 \\
\hline 2 & 6 & 6 & 6 & 6 & 6 \\
\hline 6 & 6 & 6 & 6 & 2 & 6 \\
\hline 6 & 2 & 1 & 6 & 5 & 6 \\
\hline 6 & 6 & 1 & 6 & 2 & 6 \\
\hline 5.25 & 4.75 & 3.00 & 5.88 & 3.88 & 6.00 \\
\hline
\end{tabular}

The three way Analysis of Covariance test (ANCOVA) of the Sequential group resulted in $\mathrm{F}=1.42$ and $\mathrm{p}<0.25$ which is not significant, while the three way ANCOVA test for the parallel group resulted in $\mathrm{F}=16.49$ with $\mathrm{p}<0.0001$.

The average total problem solver grade in the pre- and post-tests for both conditions are displayed in Table 3.

Table 3: A Comparison of the Average Improvement Rates

\begin{tabular}{|c|c|c|c|}
\hline \multicolumn{2}{|c|}{ Sequential Group } & \multicolumn{2}{c|}{ Parallel Group } \\
\hline $\begin{array}{c}\text { Average total } \\
\text { Pre-test grades }\end{array}$ & $\begin{array}{c}\text { Average total } \\
\text { Post-test grades }\end{array}$ & $\begin{array}{c}\text { Average total } \\
\text { Pre-test grades }\end{array}$ & $\begin{array}{c}\text { Average total } \\
\text { Post-test grades }\end{array}$ \\
\hline 7.19 & 15.88 & 13 & 15.75 \\
\hline \multicolumn{2}{|c|}{ Improved 37.15\% } & Improved 15.28\% \\
\hline
\end{tabular}

It is also worth noting that in Q2, problem solvers who received an average of 4.75 out of 6 in a pre-test scored lower in the post-test following the parallel task with an average of 3.88, which implies that they made more mistakes following the use of the system. 


\section{Discussion}

The first distinction between the two groups is the high percentage improvement of problem solvers who utilized the Sequential Module versus those who utilized the Parallel Module. This may at first make one wonder if there is no ceiling effect here since the Parallel Group started with a higher average pre-test. A ceiling effect occurs if problem solvers start with high grades in the pre-test so the number of marks that problem solver may improve in the post test becomes severely limited. In this case, it is possible that the true potential of the system is underrated if measured by percentage improvement alone. However, the reduction in the level of problem solvers in the power question (\#2) from an average of 4.75/6 to an average of 3.88/6 comes up again as a reminder that this presentation style did something that was not intended by the original design.

A sequential presentation of seven questions and solutions produces more improvement than presenting two solutions at a time for comparison with three problems in total. The complexity of the problems is closely monitored according to the definitions of complexity presented in this paper which leaves only one difference between the two presentation styles: two problems at a time for comparison versus one problem at a time.

If the geometric series problems are regarded as cars entering a compound, then the flow of one at a time is restricted by the space available for parking within the compound. However, if more than one car (problem) attempts to enter in parallel (two problems at a time for comparison) then the size of the gate matters even if the number of parking spaces available within the compound is sufficient.

Consequently, these results indicate the existence of a bottleneck at the gate of the compound (size of the "loop" and "sketchpad" memories) even though the episodic buffer has available space to hold all problems.

The second finding made by this data is shown in Table 4. The effects of similarity on the learning process are evident in the sequential group's case. The negative effect in the least similar power operations raises a question of whether similarity guides the choice of the attention of the central executive.

\begin{tabular}{|l|c|c|c|}
\hline \multicolumn{4}{|c|}{ Table 4: A Comparison of the Effects of the Difference in Similarity } \\
Pre- to Post-Test for Each Question Type \\
\hline & $\begin{array}{c}\text { Division } \\
\text { (highest similarity) }\end{array}$ & $\begin{array}{c}\text { Multiplication } \\
\text { (intermediate similarity) }\end{array}$ & $\begin{array}{c}\text { Power } \\
\text { (lowest similarity) }\end{array}$ \\
\hline $\begin{array}{l}\text { Sequential pre- to } \\
\text { post-test difference }\end{array}$ & 2.38 & 2.69 & 1.62 \\
\hline $\begin{array}{l}\text { Parallel pre- to post- } \\
\text { test difference }\end{array}$ & 3 & 0.63 & -0.87 \\
\hline
\end{tabular}

However, in spite of all this evidence a remaining criticism is that the pre-test marks for the Parallel group are much higher to start with than the Sequential group. This may mean that the total amount of improvement possible will always be lower than the improvement possible of the Sequential group. To ensure that this result had not been misread and to ensure that the pre-test scores were similar a second experiment was run.

\section{Replication Experiment}

The experiment was run in as in the evaluation experiment with one exception. The group was partitioned into two groups such that the average for each question type is similar. Students were 
sorted first according to their grades in each question and then randomly distributed to one or the other group, while continually calculating the resulting average per question. Any student who received above 5 in the pretest was excluded from continuing the experiment. Both groups had 31 problem solvers.

\section{Results}

The results obtained are shown in Table 5:

Table 5: The Results of Repeating the Experiment

\begin{tabular}{|l|c|c|c|c|c|c|}
\hline & \multicolumn{3}{|c|}{ Sequential } & \multicolumn{3}{c|}{ Parallel } \\
\hline & $\begin{array}{c}\text { Q1 } \\
\text { Multiplication }\end{array}$ & $\begin{array}{c}\text { Q2 } \\
\text { Power }\end{array}$ & $\begin{array}{c}\text { Q3 } \\
\text { Division }\end{array}$ & $\begin{array}{c}\text { Q1 } \\
\text { Multiplication }\end{array}$ & $\begin{array}{c}\text { Q2 } \\
\text { Power }\end{array}$ & $\begin{array}{c}\text { Q3 } \\
\text { Division }\end{array}$ \\
\hline Pretest & 4.61 & 4.90 & 2.00 & 4.84 & 5.00 & 2.32 \\
\hline Posttest & 4.48 & 5.61 & 4.48 & 6.00 & 2.58 & 5.97 \\
\hline $\begin{array}{l}\text { Percentage } \\
\text { Improvement }\end{array}$ & $23 \%$ & $14.5 \%$ & $124 \%$ & $24 \%$ & $-48.4 \%$ & $157 \%$ \\
\hline
\end{tabular}

These results imply that the previous results were replicated, even when the pre-test grades are similar.

\section{Conclusion}

Results indicate the existence of a bottleneck that exists in one of the stages that information goes through prior to its arrival in working memory. Prior work in limits to perceptual memory led to the development of the Broadbent (1958) Filter theory. The idea was that a filter existed in the perceptual system where items are selected for attention according to their physical traits, because their semantic traits require more processing. A number of theories eventually emerged to explain the various traits and limitations of working memory (Atkinson \& Shiffrin, 1968; Baddeley, 2001; Baddeley \& Hitch, 1974). The focus of attention therefore shifted to working memory and away from perceptual limitations (Alkhalifa, 2008b).

However, the results obtained here indicate that a limitation does exist on the information passing from the perception to working memory because if the information passed is of high bulk or complexity, then presenting it in parallel may cause a hindrance in learning.

There may be a fourth type of educational cognitive load that is dependent on the information received, which may be called bandwidth load. The term describes the extent of parallel information that a cognitive system will allow a learner to perceive without interference or loss of information. This is from an educational perspective to the working memory model that investigates the recently added module, which is the episodic buffer (Baddeley, 2000).

Such a concept informs designers of all information based systems that utilize windows or dispersed bits of information that are displayed on a computer screen. It is counterproductive to business sites, for example, to overburden a customer's cognitive bandwidth only to end up with a customer not recognizing the special bargain that is on offer during that period of time.

Further analysis shows that the memory does indeed reflect that it is episodic in nature and raises a novel question of whether or not the central executive selects what it attends to and what it neglects based on episodic similarity. Findings on analogy and similarity are also supported, be- 
cause they are likely to be occurring in the episodic buffer but the interaction between the similarity of the pre- and post-test questions requires further investigation.

There is still much more work that needs to be done to investigate the remaining characteristics of the episodic buffer. One possibility is to probe for gender differences with respect to the parallel display of information versus the serial display, especially since women achieve lower scores in mathematics than their male counterparts and this may be due to a bias by the central executive to verbal tasks.

A prior study (Alkhalifa, 2008a) found that female student levels improved significantly when they were allowed to practice programming through carefully set serial instructions. The justification that was presented was that this approach is less intimidating to them and ensures that they cover all the test cases that are expected of them. Their male colleagues may review all test cases by the process of discovery that male problem solvers enjoy engaging in with their peers. Following this finding, it may be possible that female student improvement is related to the limited reception bandwidth making serial presentation more effective than parallel presentation. Further work is necessary to estimate the full extent of the impact of these results.

\section{Acknowledgements}

I would like to thank all the eager and supportive students in the University of Bahrain for assisting and volunteering in these experiments. This project is supported by the Sh. Ebrahim Bin Hamad Fund with a total grant value of $\$ 2.5$ million.

\section{References}

Alkhalifa, E. M. (2008a). The effects of sequential programming instruction on gender differences in an IT course. IEEE Transactions on Education, 51(4), 417-422.

Alkhalifa, E. M. (2008b). Investigating limited perception effects from a cognitive science perspective. Proceedings of the Thirtieth Annual Conference of the Cognitive Science Society, Washington, D.C.

Atkinson, R. C., \& Shiffrin, R. M. (1968). Human memory: A proposed system and its control processes. In K. W. Spence \& J. T. Spence (Eds.), The psychology of learning and motivation (Vol. 2, pp. 89195). Orlando, FL: Academic Press.

Baddeley, A. D. (2003b). Working memory: Looking back and looking forward. National Review Neuroscience, 4(10), 829-839.

Baddeley, A. D. (2003a). Working memory and language: An overview. Journal of Communication Disorders, 36(3), 189-208.

Baddeley, A. D. (2001). Is working memory still working? American Psychologist, 56, 851-864.

Baddeley, A. D. (2000). The episodic buffer: A new component of working memory? Trends in Cognitive Science, 4, 417-423.

Baddeley, A. D. (1998). Working memory. Comptes Rendus de l'Académie des Sciences - Series III - Sciences de la Vie, 321(2-3), 167-173.

Baddeley, A. D., \& Hitch, G. (1974). Working memory. In G. A. Bower (Ed.), Recent advances in learning and motivation, 8, 47-90, New York: Academic Press.

Broadbent, D. E. (1958). Perception and communication. New York: Oxford University Press.

Gevers, W., Reynvoet, B., \& Fias, W. (2003). The mental representation of ordinal sequences is spatially organized. Cognition, 87(3), B87-B95. 
Gill, T. G., \& Hicks, R. (2006). Task complexity and informing science: A synthesis. Informing Science: The International Journal of an Emerging Transdiscipline, 9, 1-30. Retrieved from http://inform.nu/Articles/Vol9/v9p001-030Gill46.pdf

Guay, R. B., \& McDaniel, E. D. (1977). The relationship between mathematics achievement and spatial abilities among elementary school children. Journal for Research in Mathematics Education, 8(3), 211-215.

Miller, G. A. (1956). The magical number seven, plus or minus two: Some limits on our capacity for processing information. Psychological Review, 63, 81-97. Retrieved January 28, 2008, from http://www.musanim.com/miller1956/

Moreno, R., \& Mayer, R. E. (2000). A learner-centered approach to multimedia explanations: Deriving instructional design principles from cognitive theory. Interactive Multimedia Journal of Computer Enhanced Learning, 2(2). Retrieved January 21, 2008, from http://imej.wfu.edu/articles/2000/2/05/index.asp

Schnotz, W. \& Kurschner, C. (2007). A reconsideration of cognitive load theory. Educational Psychology Review, 19, 469-508.

Sweller, J. (1988). Cognitive load during problem solving: Effects on learning. Cognitive Science, 12, $257-$ 285.

Sweller, J. (2005). Implications of cognitive load theory for multimedia learning. In R. E. Mayer (Ed.), The Cambridge handbook of multimedia learning (pp. 19-30). New York: Cambridge University Press.

Sweller, J., Chandler, P., Tierney, P., \& Cooper, M. (1990). Cognitive load as a factor in the structuring of technical material. Journal of Experimental Psychology: General, 119, 176-192.

Sweller, J., van Merrienboer, J. J. G., \& Paas, F. G. W. C. (1998). Cognitive architecture and instructional design. Educational Psychology Review, 10(3), 251-296.

Tabbers, H. K., Martens, R. L., \& van Merriënboer, J. J. G. (2001). The modality effect in multimedia instructions. Annual conference of the Cognitive Science Society, 2001. Retrieved January 29, 2008, from http://www.hcrc.ed.ac.uk/cogsci2001/pdf-files/1024.pdf

\section{Biography}

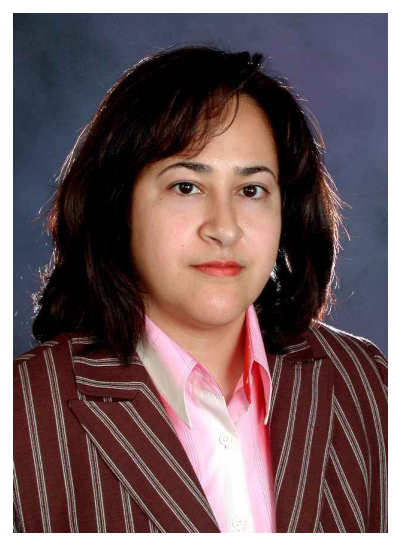

Eshaa Alkhalifa is a member of the royal family of Bahrain with over 12 years experience in University Education. She holds a PhD in Cognitive Science from the University of Edinburgh, an MSc from George Washington University, and a BSc from the University of Bahrain. She was awarded three science awards and has published in International Encyclopedias. Her main research goal is to break the ground to allow researchers to cross the divide between the purely theoretical findings of Cognitive Science and the practical applications of computerized systems, by promoting Cognitively Informed Systems. 